\title{
Simple factorization of unitary transformations
}

\author{
Hubert de Guise, ${ }^{1}$ Olivia Di Matteo, ${ }^{2,3}$ and Luis L. Sánchez-Soto ${ }^{4,5}$ \\ ${ }^{1}$ Department of Physics, Lakehead University, Thunder Bay, Ontario P7B 5E1, Canada \\ ${ }^{2}$ Department of Physics and Astronomy, University of Waterloo, Waterloo, Ontario, Canada N2L 3G1 \\ ${ }^{3}$ Institute for Quantum Computing, University of Waterloo, Waterloo, Ontario, Canada, N2L 3G1 \\ ${ }^{4}$ Departamento de Óptica, Facultad de Física, Universidad Complutense, E-28040 Madrid, Spain \\ ${ }^{5}$ Max-Planck-Institut für die Physik des Lichts, Staudtstraße 2, D-91058 Erlangen, Germany
}

(Received 7 August 2017; published 20 February 2018)

\begin{abstract}
We demonstrate a method for general linear optical networks that allows one to factorize any SU(n) matrix in terms of two $\mathrm{SU}(n-1)$ blocks coupled by an SU(2) entangling beam splitter. The process can be recursively continued in a straightforward way, ending in a tidy arrangement of SU(2) transformations. The method hinges only on a linear relationship between input and output states, and can thus be applied to a variety of scenarios, such as microwaves, acoustics, and quantum fields.
\end{abstract}

DOI: 10.1103/PhysRevA.97.022328

\section{INTRODUCTION}

Linear optics constitutes an outstanding setting for information processing. The Knill-Laflamme-Milburn [1] protocol for scalable quantum computing, experimental boson sampling [2-5], or the generation of quantum random walks [6-11], are good examples of how the growing capabilities of fabrication technologies are transforming the field of quantum photonics [12]. In addition, these capabilities are altering classical areas, such as microwave photonics [13] or optical networking [14,15].

A basic ingredient for all these developments is the design of reconfigurable setups that can perform any linear operation. The influential work by Reck et al. [16], which can be traced back to the elegant results of Murnaghan [17], established that a specific array of basic two-mode operations is sufficient to implement any unitary in $\mathrm{U}(n)$. In this way, it is indeed possible to construct a single device with ample versatility to implement any possible unitary operation up to the specified number of modes. Recently, demonstrations of large-scale linear networks have appeared [18,19].

Continued interest in these universal processors for classical and quantum applications has led to new designs [20,21]. In particular, an intriguing proposal came out [22] requiring roughly half the optical depth of the original Reck et al. design [16]. This is important for minimizing optical losses and reducing fabrication resources.

We discuss here a decomposition of any $n \times n$ unitary in terms of two $(n-1) \times(n-1)$ unitaries coupling the same $n-1$ modes, and a single $2 \times 2$ unitary coupling one of those $n-1$ to the remaining mode [23]. The scheme is recursive; it can be halted at any dimensionality of subtransformations or performed in its end, resulting in a tidy arrangement of SU(2) gadgets. The structure is thus

$$
R^{n}(\Omega)=R^{n-1}(\tilde{\Omega}) R_{12}(\alpha, \beta, \alpha) R^{n-1}\left(\tilde{\Omega}^{\prime}\right) .
$$

This factorization is economical from a computational perspective: It requires the evaluation of fewer matrices than that of Reck et al. [16] and this advantage increases with $n$. This economy is particularly relevant as multiparticle scattering by large unitary arrays are now within the realm of experimental feasibility. Finally, with the transformations $R^{n-1}(\tilde{\Omega})$ and $R^{n-1}\left(\tilde{\Omega}^{\prime}\right)$ in the same subgroup, the scheme is well adapted to calculations using the Gelfan'd-Tseitlin machinery [24-27].

We demonstrate the universality of the design and explain in detail some pertinent examples that reveal the directness of the procedure.

As a byproduct, the Haar measure of $\mathrm{U}(n)$ can easily be factorized according to our scheme. There is a fresh interest in realizing Haar random unitary matrices [28], because of the important role they play in various tasks for quantum cryptography [29] and quantum protocols [30]. From this viewpoint, our analysis, which is reminiscent of the ideas sketched in Ref. [23], might be instrumental for a simpler implementation of these operations [31,32].

Finally, it is important to note that, while our scheme is generally versatile, applies to any $n$, and can be used for arbitrary representations of $\mathrm{SU}(n)$, there exist other algorithms in dimension $2^{m}$ (see, e.g., Refs. [33-35]) that achieve more efficient decompositions with respect to the quantum circuit model. Our decomposition does not improve on the bounds presented in this other work, but instead offers a convenient and experiment-driven parametrization that retains the same scaling with $n$ regardless of the internal tensor-product structure of the system.

\section{RECURSIVE FACTORIZATION OF UNITARY TRANSFORMATIONS}

An ideal, lossless linear optical circuit with $n$ input channels and $n$ output channels performs an optical transformation which can be described by an $n \times n$ matrix; i.e., it belongs to the group $\mathrm{U}(n)$. We can always factor an overall phase to make the determinant equal to 1 , so we deal with $\mathrm{SU}(n)$ [17], which has $n^{2}-1$ independent parameters. 
Our goal is to explore an intuitive factorization of $\mathrm{SU}(n)$ transformations, which is especially germane for our purposes here and has the additional advantage of being highly recursive. To be more precise, our method can be symbolically stated in the following way: Any $R^{n}(\Omega) \in \mathrm{SU}(n)$ can be written as in Eq. (1), where $R^{n-1}(\tilde{\Omega}), R^{n-1}\left(\tilde{\Omega}^{\prime}\right) \in \mathrm{SU}(n-1)$. Here, $R_{i j}$ is a matrix of the form,

$$
R_{i j}=\left(\begin{array}{ccccccc}
1 & 0 & \ldots & \ldots & \ldots & \ldots & 0 \\
0 & 1 & & & & & \vdots \\
\vdots & & \ddots & & & & \vdots \\
\vdots & & & \mathscr{R}_{i j} & & & \vdots \\
\vdots & & & & \ddots & & \vdots \\
\vdots & & & & & 1 & 0 \\
0 & \ldots & \ldots & \ldots & \ldots & 0 & 1
\end{array}\right),
$$

coupling adjacent modes $i$ and $j$ (with $j=i+1$ ) via an $\mathrm{SU}(2)$ transformation $\mathscr{R}_{i j}(\alpha, \beta, \gamma)$ acting on them.

We recall that any $\mathscr{R}(\alpha, \beta, \gamma) \in \mathrm{SU}(2)$, parametrized by the Euler angles, can be always written as

$$
\begin{aligned}
\mathscr{R}(\alpha, \beta, \gamma)= & \mathscr{R}_{z}(\alpha) \mathscr{R}_{y}(\beta) \mathscr{R}_{z}(\gamma)=\left(\begin{array}{cc}
e^{i \alpha / 2} & 0 \\
0 & e^{-i \alpha / 2}
\end{array}\right) \\
& \times\left(\begin{array}{cc}
\cos (\beta / 2) & -\sin (\beta / 2) \\
\sin (\beta / 2) & \cos (\beta / 2)
\end{array}\right)\left(\begin{array}{cc}
e^{i \gamma / 2} & 0 \\
0 & e^{-i \gamma / 2}
\end{array}\right),
\end{aligned}
$$

where we follow the standard notation of Ref. [36]. This factorization is in turn a prescription for how to construct the SU(2) device: When the information is encoded in the polarization, a set of three wave plates is enough [37]; for path encoding, this can be mapped to a beam splitter of transmittance $\cos ^{2}(\beta / 2)$ and phase shift $\gamma$, plus a phase shifter that gives the required extra phase $\alpha$. The action of $R_{i j}$ can also be devised for more complex systems, such as ion traps [38] and superconducting circuits [39].

Let us illustrate our scheme in a constructive way, starting with the simplest case of SU(3). Of course, other parametrizations of SU(3) elements are possible [40-42], but one that is particularly useful [43] is into a sequence of adjacent $\mathrm{SU}(2)_{i+1}$ transformations mixing channels $i$ and $i+1$. More explicitly, with $R^{3}(\Omega) \in \mathrm{SU}(3)$, we have

$$
R^{3}(\Omega)=R_{23}\left(\alpha_{1}, \beta_{1}, \gamma_{1}\right) R_{12}\left(\alpha_{2}, \beta_{2}, \alpha_{2}\right) R_{23}\left(\alpha_{3}, \beta_{3}, \gamma_{3}\right) .
$$

The middle transformation in the sequence depends only on two parameters (so, it is just a pure beam splitter), and the whole $R^{3}(\Omega)$ depends on eight, as it should. This factorization is symbolically denoted by a sequence of $2 \times 2$ squares representing SU(2) transformations, as illustrated in Fig. 1. To lighten the notation, we write $R_{i j}(k)$ where $k$ denotes the number of parameters in the transformation. For example,

$$
R_{i j}(2):=R_{i j}(\alpha, \beta, \alpha), \quad R_{i j}(3):=R_{i j}(\alpha, \beta, \gamma)
$$

is used throughout. In addition, the parameters in the first and last $R_{23}$ operations are understood to be different even

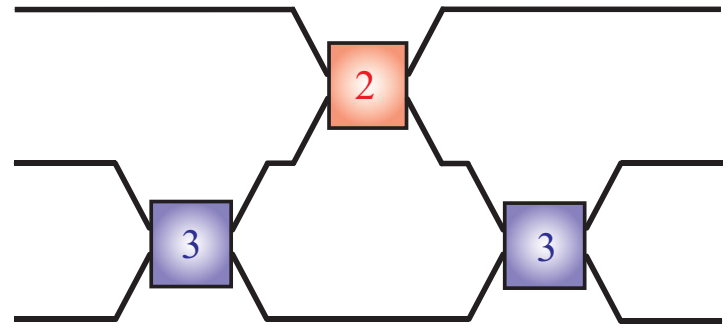

FIG. 1. A schematic illustration of the factorization of an SU(3) transformation into a sequence of SU(2) transformations. Each mode is represented by a line. Transformations between modes are represented by boxes, into which the modes are fed. The number on each box indicates the number of parameters in the transformation; we use color for visual ease of distinguishing between transformations on the same number of modes, but differing numbers of parameters.

if this is not indicated in the boxes. For completeness we recall that finite transformations of the $R_{i j}$ type are obtained by exponentiation of generator matrix elements:

$$
R_{i j}(\alpha, \beta, \gamma)=e^{-i \frac{\alpha}{2}\left(C_{i i}-C_{j j}\right)} e^{-\frac{\beta}{2}\left(C_{i j}-C_{j i}\right)} e^{-i \frac{\gamma}{2}\left(C_{i i}-C_{j j}\right)},
$$

where $C_{i j}$, with $i, j=1, \ldots n$, are generators of $\mathrm{U}(n)$ mixing modes $(i j)$ when $i \neq j$ or measuring the population $i$ when $i=j$.

To proceed further, we next factorize an SU(4) matrix. We start with a $4 \times 4$ special unitary matrix $M$ which we write generically as

$$
M=\left(\begin{array}{cccc}
x & * & * & * \\
y & * & * & * \\
z & * & * & * \\
w & * & * & *
\end{array}\right)
$$

Apply $R_{34}^{-1}\left(\alpha_{1}, \beta_{1}, \gamma_{1}\right)$ indicated in Eq. (2), namely

$$
R_{34}^{-1}\left(\alpha_{1}, \beta_{1}, \gamma_{1}\right)=\left(\begin{array}{cc}
\mathbb{1}_{2 \times 2} & 0_{2 \times 2} \\
0_{2 \times 2} & \mathscr{R}_{34}^{-1}\left(\alpha_{1}, \beta_{1}, \gamma_{1}\right)
\end{array}\right) .
$$

Choose now the Euler angles as

$$
\begin{aligned}
e^{-\frac{1}{2} i\left(\alpha_{1}+\gamma_{1}\right)} \cos \left(\frac{1}{2} \beta_{1}\right) & =\frac{z}{\sqrt{1-|x|^{2}-|y|^{2}}}, \\
e^{-\frac{1}{2} i\left(\alpha_{1}-\gamma_{1}\right)} \sin \left(\frac{1}{2} \beta_{1}\right) & =\frac{w}{\sqrt{1-|x|^{2}-|y|^{2}}},
\end{aligned}
$$

to obtain

$$
R_{34}^{-1}\left(\alpha_{1}, \beta_{1}, \gamma_{1}\right) M=\left(\begin{array}{cccc}
x & * & * & * \\
y & * & * & * \\
\sqrt{1-|x|^{2}-|y|^{2}} & * & * & * \\
0 & * & * & *
\end{array}\right),
$$

that is, we make a 0 appear at position $(4,1)$. The second step 

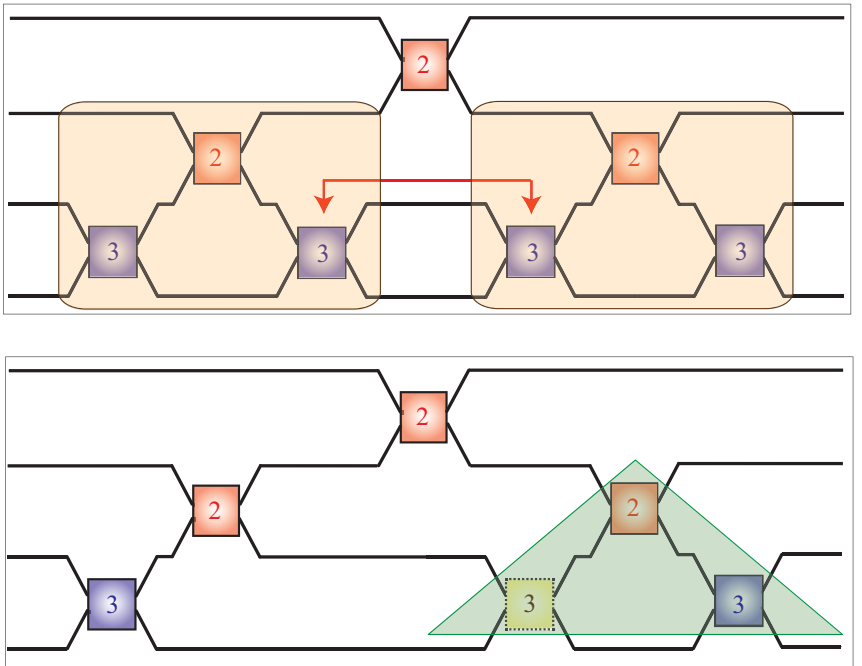

FIG. 2. A schematic illustration of the factorization of an SU(4) transformation as a sequence of $\mathrm{SU}(3)$ transformations, each itself written as $\mathrm{SU}(2)$ blocks.

is to apply $R_{23}^{-1}$ to make a 0 appear at position $(3,1)$, and finally $R_{12}$ to produce a 0 in position $(2,1)$ :

$$
\begin{gathered}
R_{12}^{-1} R_{23}^{-1} R_{34}^{-1} M=\left(\begin{array}{llll}
1 & 0 & 0 & 0 \\
0 & * & * & * \\
0 & * & * & * \\
0 & * & * & *
\end{array}\right), \\
M=R_{34} R_{23} R_{12}\left(\begin{array}{llll}
1 & 0 & 0 & 0 \\
0 & * & * & * \\
0 & * & * & * \\
0 & * & * & *
\end{array}\right),
\end{gathered}
$$

with the phases chosen so that 1 occurs in position $(1,1)$. Since $\sum_{j=1}^{4}\left|a_{i j}\right|^{2}=1$ for any row of a unitary matrix, the last step also forces $0 \mathrm{~s}$ on the first row. As all the $R_{i j}$ s are unitary, the result of $\mathscr{R}_{12}^{-1} \mathscr{R}_{23}^{-1} \mathscr{R}_{34}^{-1}$ acting on the original matrix is a $3 \times 3$ unitary submatrix, for which the original decomposition in Eq. (4) can be applied.

Parameter counting (after suitable relabeling of the modes) can be neatly understood graphically. First, consider an SU(4) transformation obtained from an $\mathrm{SU}(2)$ one of the type $R_{12}(\alpha, \beta, \alpha)$, sandwiched between two $\mathrm{SU}(3)$ transformations, as illustrated in Fig. 2. Each SU(3) transformation is of the type given in Fig. 1, and they are indicated by shaded squares.

Closer inspection of Fig. 2 shows that there are two adjacent $R_{34}$, joined by a red arrow, that commute with the middle $R_{12}$, as they mix completely disjoint channels. One can thus "push together" or combine these transformations, as they are of the same $\mathrm{SU}(2)$ type, so their combination is a single $\mathrm{SU}(2)$ matrix of the $R_{34}$ type. This is symbolically indicated by a box of different color. The resulting system is in a green shaded triangle, which represents just a full SU(3) transformation. The total number of parameters is 15 , as it should be. Moreover, as a result of pushing together boxes, the partial SU(3) sequence
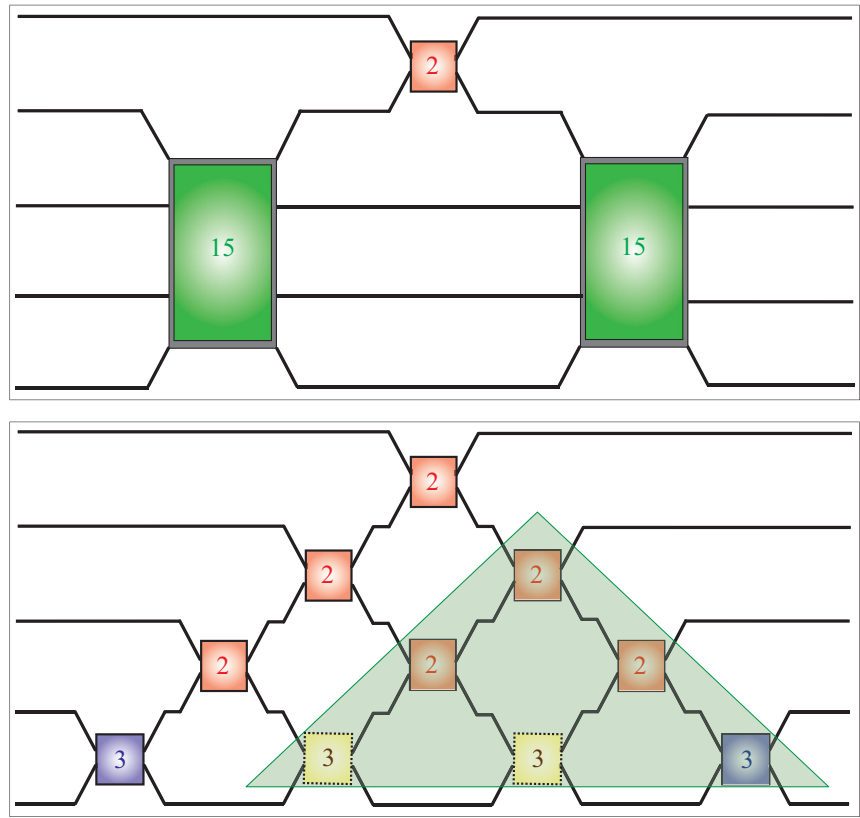

FIG. 3. A schematic illustration of the factorization of an SU(5) transformation as a sequence of two SU(4) transformations, coupled by an SU(2). The bottom panel shows the final result in terms of SU(2) blocks. The shaded triangle is an equivalent SU(4) transformation.

$R_{23} R_{34}$ in Eq. (12) is an SU(3)/SU(2) transformation obtained from Eq. (4) by setting the second $R_{23}$ to $\mathbb{1}$.

We can now immediately generalize the scheme to construct an SU(5) transformation as an SU(2) sandwiched in between two SU(4) transformations, represented as green boxes (with 15 parameters) in Fig. 3. Again, boxes can be combined into a single SU(3) following the same pushing procedure. The final result is just a SU(4) transformation (indicated again by the shaded triangle) and the total number of parameters is 24 . We have written a PYTHON software package capable of generating the entire set of parameters, which we make available online [44].

At this time it would be useful to compare our decomposition to other existing schemes. We recall that factorizations are representation independent: Even if a scheme is found using the fundamental $n \times n$ representation of $\mathrm{SU}(n)$, it remains valid for any other representation of $\mathrm{SU}(n)$. Any general $\mathrm{SU}(n)$ transformation must also depend on $n^{2}-1$ parameters: The number of exponentiations in any scheme must always amount to $n^{2}-1$ else the transformation is not general.

In Fig. 4 we illustrate the designs of Reck et al. [16] and Clements et al. [22] for four modes. Both exclusively employ two-parameter SU(2) transformations; i.e., the mesh is made only of beam splitters. The single-mode phase shifts are programmed at the output of the channels. This is in contradistinction with our results displayed in Fig. 2.

The decomposition of Reck et al. [16] uses transformations on both adjacent and nonadjacent modes, for which the evaluation of $R_{i j}$ transformations for every possible $(i j)$ pair of the network is necessary. The scheme is recursive with $\mathrm{SU}(n-1)$ transformations easily identifiable as a subblock of the full $\mathrm{SU}(n)$. 


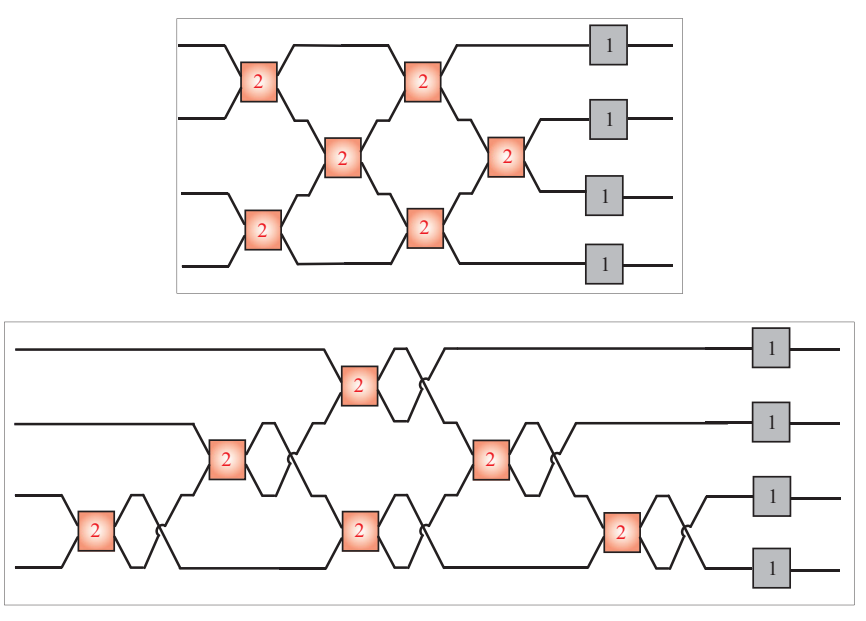

FIG. 4. Illustration of the factorization schemes of Clements et al. [22] (top) and Reck et al. [16] for the case of four modes.

Our scheme is also recursive, but with the same type of $\mathrm{SU}(n-1)$ transformations appearing twice in Eq. (1), and mixes only adjacent modes. It achieves computational economy over Reck et al. [16] because some generators are used multiple times, so that fewer of them need to be computed. For instance, our SU(4) transformation uses a (34) block three times, (23) twice, and (12) once (in general, $R_{i i+1}$ is used $i$ times so $C_{i+1}$ and its transpose conjugate are used $i$ times).

Then our scheme requires nine types of matrix elements: six of the type $C_{34}, C_{43}, C_{23}, C_{32}, C_{22}, C_{33}, C_{44}$ for $\mathrm{SU}(3)$ transformations of modes (234), plus three more $C_{12}, C_{21}, C_{11}$ for the SU(2) transformation of modes (12). Taking into account the fact that $C_{j i}=C_{i j}^{T}$, this generalizes to $n-1$ matrices of the type $C_{i i+1}$ and $n$ diagonal matrices $C_{i i}$ for $\mathrm{SU}(n)$. Reck et al., on the other hand, require the evaluation of three additional matrices for the nonadjacent transformations of the type $R_{13}, R_{14}$, or $R_{24}$, which entails the computation of $\frac{1}{2}\left(n^{2}-n\right)$ generators of the $C_{i j}$ type with $j>i$, and $n$ diagonal matrices $C_{i i}$ for $\mathrm{SU}(n)$. Our scheme thus saves the evaluation of $\frac{1}{2}(n-1)(n-2)$ generators over Reck et al. [16], with the additional advantage that the associated scaling in the number of $C_{i+1}$ needed is linear rather than polynomial. In fact one can see that, by reusing $(i, i+1)$ blocks, our scheme minimizes the number of matrix elements to be computed, as one cannot construct a general transformation by using fewer types of blocks.

This economy becomes very relevant in large networks containing many particles, as the following pertinent example confirms. Consider the scattering of $p$ indistinguishable photons by an $n \times n$ interferometer. This system, currently very popular in the context of boson sampling, is described by an $\left(\begin{array}{c}n+p-1 \\ p\end{array}\right)$-dimensional representation of $\mathrm{SU}(n)$ obtained by exponentiating generators using the same factorization as the fundamental $n \times n$ representation, with each $\mathrm{SU}(n-1)$ a block diagonal submatrix. Thus for $n=9$ and $p=5$ [45], one must exponentiate a sequence of matrices of size $1287 \times 1287$. Permanents are entries of the full $1287 \times 1287$ matrix; i.e., $D$ functions for this irreducible representation [46]. Whereas the decomposition of Reck et al. [16] (or its primal version by
Murnaghan [23]) requires the evaluation of 36 nondiagonal $C_{i j}$ with $j>i$, their transpose conjugates, and nine $C_{i i}$, our scheme requires the evaluation of only eight $C_{i i+1}$ matrices, their transpose conjugates, and nine $C_{i i}$. As the size of practical interferometers increases, the linear scaling of this scheme thus stands to offer substantial computational savings. For boson sampling, where the number of modes $n$ is ideally expected to scale like the square of the number $p$ of photons, the matrices of the symmetric representation are of size $\sim 10^{5} \times 10^{5}$ for $p=5$. Clearly, minimizing the number of $C_{i j}$ to evaluate becomes an issue important from a resource and accuracy perspective.

Note that the factorization of Eq. (1) is also very natural as the canonical set of basis states, enumerated in terms of Gelfan'd-Tseitlin patterns $\left|(m)_{n}\right\rangle$, also follow the $\mathrm{SU}(n) \downarrow$ $\mathrm{SU}(n-1)$ subgroup chain [24-27]. Thus the group functions,

$$
\left\langle(m)_{n}\left|R^{n-1}(\tilde{\Omega}) R_{12}(\alpha, \beta, \alpha) R^{n-1}\left(\tilde{\Omega}^{\prime}\right)\right|\left(m^{\prime}\right)_{n}\right\rangle,
$$

are naturally expressed as a sum of products of $\mathrm{SU}(n-1) \times$ $\mathrm{SU}(2) \times \mathrm{SU}(n-1)$ group functions. A byproduct of this form is that the $\mathrm{SU}(n-1)$ subgroup transformations are blockdiagonal in the Gelfan'd-Tseitlin basis, a useful feature to check calculations.

The scheme of Clements et al. [22] has a different structure, corresponding instead to a rectangular mesh of beam splitters. One might expect the triangular scheme to be more resilient to losses in experiments in which only a small proportion of its input ports are accessed, whereas the rectangular scheme is likely to be beneficial for experiments that involve accessing most of its inputs.

Algorithmically, our scheme differs from the scheme of Clements et al. [22] in the order in which 0s are made to appear when working on the original matrix $M$. As a result (and by design), the scheme mixes channels "as early as possible" and achieves depth of $n$. In contradistinction our scheme mixes channels "as late as possible": This is necessary to achieve the highly recursive factorization structure of Eq. (1), but the tradeoff is a scheme of depth $2 n-3$, on par with Reck et al. [16].

This difference in optical depth is the reason why, in a simple loss model that assumes equal insertion loss for every beam splitter, Clements et al. [22] always has better performance. A careful analysis can be found in Ref. [22]. In other words, in Clements et al. [22] all the modes encounter roughly the same number of beam splitters; in the triangle, transformation $R_{i j}$ occurs $i$ times, then modes experiencing more beam splitters experience more loss and so the lower modes get more scrambled than those at the top of the triangle.

Finally, we stress that in our scheme the rightmost $R^{n-1}$ transformation is a full subgroup transformation, while the leftmost is a partial subgroup transformation. Pushing and combining boxes show how an $\mathrm{SU}(n)$ device can be constructed from two $\mathrm{SU}(n-1)$ devices and a single $\mathrm{SU}(2)$ device. In this respect, it is worth mentioning that the recent interest in networks of multiport devices instead or beam splitters $[47,48]$ makes our algorithm especially relevant, as we can decompose a unitary as coupled $\mathrm{SU}(d)$ devices, with $d$ chosen at will. This makes also the difference with the well-known decompositions of quantum gates $[35,49,50]$. 


\section{RECURSIVE HAAR MEASURES}

The recursive factorization in Eq. (1) also implies a recursive form of the Haar measure. We just briefly recall that a Haar measure is an invariant measure on the group manifold. It thus provides a natural probability distribution over the group, in the sense that it equally weighs different regions, thus behaving like a uniform distribution on $\mathrm{SU}(n)$. This is of utmost importance for the generation of statistical ensembles of unitary matrices [28], which is a useful tool in many fields of physics, as heralded in the Introduction.

For SU(2) we have

$$
d \Omega_{2}=\sin \beta d \beta d \alpha d \gamma
$$

Simple application of the usual method yields [51] the $\mathrm{SU}(3)$ measure, namely,

$$
d \Omega_{3}=d \Omega_{2}(1)\left[\sin \beta_{2} \sin ^{2}\left(\frac{1}{2} \beta_{2}\right) d \alpha_{2} d \beta_{2}\right] d \Omega_{2}(3),
$$

with $d \Omega_{2}(k)=\sin \beta_{k} d \beta_{k} d \alpha_{k} d \gamma_{k}$ an $\mathrm{SU}(2)$ measure.

For SU(4), we find

$$
d \Omega_{4}=d \tilde{\Omega}_{3}(1,2)\left[\sin ^{4}\left(\frac{1}{2} \beta_{3}\right) \sin \beta_{3}\right] d \Omega_{3}(4,5,6),
$$

where $d \Omega_{3}(i, j, k)$ is an $\mathrm{SU}(3)$ measure of the arguments in parentheses and

$$
d \tilde{\Omega}_{3}(1,2)=d \Omega_{2}(1)\left[\sin \beta_{2} \sin ^{2}\left(\frac{1}{2} \beta_{2}\right) d \alpha_{2} d \beta_{2}\right]
$$

is a coset measure, with fewer parameters compared to the full measure. The effect of combining $R_{34}$ transformations by pushing an $R_{34}$ transformation under $R_{12}$, which we discussed in the previous section, results in the removal of one $d \Omega_{2}$ factor in $d \tilde{\Omega}_{3}(1,2)$.

In SU(5) we find

$$
d \Omega_{5}=d \tilde{\Omega}_{4}(1,2,3)\left[\sin ^{6}\left(\frac{1}{2} \beta_{4}\right) \sin \beta_{4}\right] d \Omega_{4}(5,6,7,8,9,10),
$$

with $d \Omega_{4}$ and $d \tilde{\Omega}_{4}(1,2,3)$ having the same meaning as before. The recursion steps to higher $n$ are clear. Quite clearly the middle factor is conveniently found to be of the form,

$$
\sin \beta_{n-1} \sin ^{2(n-2)}\left(\frac{1}{2} \beta_{n-1}\right),
$$

with maximum at $\cos \beta_{n-1}=-(n-2) /(n-1)$. This is in agreement with the result of [23] and other results obtained from different perspectives [32,52] and it is very useful in many instances, e.g., for the parametrization of the families of most probable matrices.

The parametrization of Eq. (1) and the examples above also neatly illustrate how to isolate from the full measure the coset measure $d \tilde{\Omega}_{n-1}$ over $\mathrm{SU}(n) / \mathrm{U}(n-1)$ : It is obtained by removing the full $\mathrm{SU}(n-1)$ part containing $(n-1)^{2}-1$ factors from full measure. The usefulness of this coset measure comes from applications to coherent states [53]; these states "live" in the coset space $\mathrm{SU}(n) / \mathrm{U}(n-1)$ so the coset measure is what is required for integration over these states.

\section{CONCLUDING REMARKS}

In conclusion, we have discussed the design for universal linear $n \times n$ optical networks which arises very naturally by recycling as much as possible the elements already present in a network of size $(n-1) \times(n-1)$. Our algorithm decomposes unitary matrices into a sequence of unitary matrices of one dimension less, entangled by a beam splitter. We expect that our compact method will play an important role in the development of optical processors for both classical and quantum applications.

In a more technical context, our method allows one to write $\mathrm{SU}(n)$ group functions in terms of $\mathrm{SU}(n-1)$ group functions, thereby extending the result of Ref. [43] and the parametrization of coherent states in $\mathrm{SU}(n) / \mathrm{U}(m)$ for arbitrary representations of $\mathrm{SU}(n)$ when the highest weight state is $\mathrm{U}(m)$ invariant. Work along these lines is now in progress.

\section{ACKNOWLEDGMENTS}

The work of H.d.G. and O.D.M. is supported by NSERC of Canada. I.Q.C. is supported in part by the Government of Canada and the Province of Ontario. O.D.M. is also grateful for hospitality at the MPL. L.L.S.S. acknowledges financial support from the Spanish MINECO (Grant No. FIS201567963-P). We thank L. Rudnicki for helpful discussions.
[1] E. Knill, R. Laflamme, and G. J. Milburn, A scheme for efficient quantum computation with linear optics, Nature (London) 409, 46 (2001).

[2] J. B. Spring, B. J. Metcalf, P. C. Humphreys, W. S. Kolthammer, X.-M. Jin, M. Barbieri, A. Datta, N. Thomas-Peter, N. K. Langford, D. Kundys, J. C. Gates, B. J. Smith, P. G. R. Smith, and I. A. Walmsley, Boson sampling on a photonic chip, Science 339, 798 (2013).

[3] M. A. Broome, A. Fedrizzi, S. Rahimi-Keshari, J. Dove, S. Aaronson, T. C. Ralph, and A. G. White, Photonic boson sampling in a tunable circuit, Science 339, 794 (2013).

[4] A. Crespi, R. Osellame, R. Ramponi, D. J. Brod, E. F. Galvão, N. Spagnolo, C. Vitelli, E. Maiorino, P. Mataloni, and F. Sciarrino, Integrated multimode interferometers with arbitrary designs for photonic boson sampling, Nat. Photon. 7, 545 (2013).
[5] M. Tillmann, B. Dakic, R. Heilmann, S. Nolte, A. Szameit, and P. Walther, Experimental boson sampling, Nat. Photon. 7, 540 (2013).

[6] B. Do, M. L. Stohler, S. Balasubramanian, D. S. Elliott, C. Eash, E. Fischbach, M. A. Fischbach, A. Mills, and B. Zwickl, Experimental realization of a quantum quincunx by use of linear optical elements, J. Opt. Soc. Am. B 22, 499 (2005).

[7] H. B. Perets, Y. Lahini, F. Pozzi, M. Sorel, R. Morandotti, and Y. Silberberg, Realization of Quantum Walks with Negligible Decoherence in Waveguide Lattices, Phys. Rev. Lett. 100, 170506 (2008).

[8] A. Peruzzo, M. Lobino, J. C. F. Matthews, N. Matsuda, A. Politi, K. Poulios, X.-Q. Zhou, Y. Lahini, N. Ismail, K. Wörhoff, Y. Bromberg, Y. Silberberg, M. G. Thompson, and J. L. O'Brien, Quantum walks of correlated photons, Science 329, 1500 (2010). 
[9] M. A. Broome, A. Fedrizzi, B. P. Lanyon, I. Kassal, A. AspuruGuzik, and A. G. White, Discrete Single-Photon Quantum Walks with Tunable Decoherence, Phys. Rev. Lett. 104, 153602 (2010).

[10] A. Schreiber, A. Gábris, P. P. Rohde, K. Laiho, M. Štefaňák, V. Potoček, C. Hamilton, I. Jex, and C. Silberhorn, A 2D quantum walk simulation of two-particle dynamics, Science 336, 55 (2012).

[11] Z.-H. Bian, J. Li, X. Zhan, J. Twamley, and P. Xue, Experimental implementation of a quantum walk on a circle with single photons, Phys. Rev. A 95, 052338 (2017).

[12] J. Carolan, C. Harrold, C. Sparrow, E. Martín-López, N. J. Russell, J. W. Silverstone, P. J. Shadbolt, N. Matsuda, M. Oguma, M. Itoh, G. D. Marshall, M. G. Thompson, J. C. F. Matthews, T. Hashimoto, J. L. O'Brien, and A. Laing, Universal linear optics, Science 349, 711 (2015).

[13] J. Capmany, I. Gasulla, and D. Perez, Microwave photonics: The programmable processor, Nat. Photon. 10, 6 (2016).

[14] L. Chen, E. Hall, L. Theogarajan, and J. Bowers, Photonic switching for data center applications, IEEE Photon. J. 3, 834 (2011).

[15] R. Stabile, A. Albores-Mejia, A. Rohit, and K. A. Williams, Integrated optical switch matrices for packet data networks, Microsyst. Nanoeng. 2, 15042 (2016).

[16] M. Reck, A. Zeilinger, H. J. Bernstein, and P. Bertani, Experimental Realization of any Discrete Unitary Operator, Phys. Rev. Lett. 73, 58 (1994).

[17] F. D. Murnaghan, The Unitary and Rotation Groups (Spartan, Washington, DC, 1962).

[18] N. Harris, D. Bunandar, M. Pant, G. R. Steinbrecher, J. Mower, M. Prabhu, T. Baehr-Jones, M. Hochberg, and D. Englund, Large-scale quantum photonic circuits in silicon, Nanophotonics 5, 456 (2016).

[19] N. C. Harris, G. R. Steinbrecher, M. Prabhu, Y. Lahini, J. Mower, D. Bunandar, C. Chen, F. N. C. Wong, T. Baehr-Jones, M. Hochberg, S. Lloyd, and D. Englund, Quantum transport simulations in a programmable nanophotonic processor, Nat. Photon. 11, 447 (2017).

[20] D. A. B. Miller, Perfect optics with imperfect components, Optica 2, 747 (2015).

[21] I. Dhand and S. K. Goyal, Realization of arbitrary discrete unitary transformations using spatial and internal modes of light, Phys. Rev. A 92, 043813 (2015).

[22] W. R. Clements, P. C. Humphreys, B. J. Metcalf, W. S. Kolthammer, and I. A. Walmsley, Optimal design for universal multiport interferometers, Optica 3, 1460 (2016).

[23] F. D. Murnaghan, The element of volume of the rotation group, Proc. Natl. Acad. Sci. USA 38, 69 (1952).

[24] I. M. Gelfand and M. L. Zetlin, Finite-dimensional representations of the group of unimodular matrices, Dokl. Akad. Nauk SSSR 71, 825 (1950).

[25] I. M. Gelfand, Representations of the Rotation and Lorentz Groups and their Applications (Pergamon, London, 1963).

[26] A. Alex, M. Kalus, A. Huckleberry, and J. von Delft, A numerical algorithm for the explicit calculation of $\mathrm{SU}(n)$ and $\mathrm{SL}(N, C)$ Clebsch-Gordan coefficients, J. Math. Phys. 52, 023507 (2011).

[27] J. D. Louck, Recent progress toward a theory of tensor operators in the unitary groups, Am. J. Phys. 38, 3 (1970).

[28] K. Życzkowski and M. Kuś, Random unitary matrices, J. Phys. A: Math. Gen. 27, 4235 (1994).
[29] P. Hayden, D. Leung, P. W. Shor, and A. Winter, Randomizing quantum states: Constructions and applications, Commun. Math. Phys. 250, 371 (2004).

[30] A. Abeyesinghe, I. Devetak, P. Hayden, and A. Winter, The mother of all protocols: restructuring quantum information's family tree, Proc. Roy. Soc. A 465, 2537 (2009).

[31] C. Spengler, M. Huber, and B. C. Hiesmayr, Composite parameterization and Haar measure for all unitary and special unitary groups, J. Math. Phys. 53, 013501 (2012).

[32] N. J. Russell, L. Chakhmakhchyan, J. L. O'Brien, and A. Laing, Direct dialling of Haar random unitary matrices, New J. Phys. 19, 033007 (2017).

[33] V. V. Shende, S. S. Bullock, and I. L. Markov, Synthesis of quantum-logic circuits, IEEE Trans. Comput.-Aided Design Integr. Circuits Syst. 25, 1000 (2006).

[34] M. Möttönen, J. J. Vartiainen, V. Bergholm, and M. M. Salomaa, Quantum Circuits for General Multiqubit Gates, Phys. Rev. Lett. 93, 130502 (2004).

[35] J. J. Vartiainen, M. Möttönen, and M. M. Salomaa, Efficient Decomposition of Quantum Gates, Phys. Rev. Lett. 92, 177902 (2004).

[36] D. A. Varshalovich, A. N. Moskalev, and V. K. Khersonskii, Quantum Theory of Angular Momentum (World Scientific, Singapore, 1988).

[37] R. Simon and N. Mukunda, Universal SU(2) gadget for polarization optics, Phys. Lett. A 138, 474 (1989).

[38] C. Shen, Z. Zhang, and L. M. Duan, Scalable Implementation of Boson Sampling with Trapped Ions, Phys. Rev. Lett. 112, 050504 (2014).

[39] B. Peropadre, G. G. Guerreschi, J. Huh, and A. Aspuru-Guzik, Proposal for Microwave Boson Sampling, Phys. Rev. Lett. 117, 140505 (2016).

[40] D. Chang and P. B. Pal, Orthogonal and unitary matrices in arbitrary dimensions, Phys. Rev. D 32, 3294 (1985).

[41] M. Byrd and E. C. G. Sudarshan, SU(3) revisited, J. Phys. A: Math. Gen. 31, 9255 (1998).

[42] P. A. Ivanov, E. S. Kyoseva, and N. V. Vitanov, Engineering of arbitrary $\mathrm{U}(n)$ transformations by quantum Householder reflections, Phys. Rev. A 74, 022323 (2006).

[43] D. J. Rowe, B. C. Sanders, and H. de Guise, Representations of the Weyl group and Wigner functions for SU(3), J. Math. Phys. 40, 3604 (1999).

[44] https://github.com/glassnotes/Caspar.

[45] H. Wang, Y. He, Y.-H. Li, Z.-E. Su, B. Li, H.-L. Huang, X. Ding, M.-C. Chen, C. Liu, Jian Qin, J.-P. Li, Y.-M. He, C. Schneider, M. Kamp, C.-Z. Peng, S. Höfling, C.-Y. Lu, and J.-W. Pan, Highefficiency multiphoton boson sampling, Nat. Photon. 11, 361 (2017).

[46] H. de Guise, D. Spivak, J. Kulp, and I. Dhand, D-functions and immanants of unitary matrices and submatrices, J. Phys. A: Math. Theor. 49, 09LT01 (2016).

[47] D. S. Simon, C. A. Fitzpatrick, and A. V. Sergienko, Group transformations and entangled-state quantum gates with directionally unbiased linear-optical multiports, Phys. Rev. A 93, 043845 (2016).

[48] D. S. Simon, C. A. Fitzpatrick, S. Osawa, and A. V. Sergienko, Quantum simulation of discrete-time Hamiltonians using directionally unbiased linear optical multiports, Phys. Rev. A 95, 042109 (2017). 
[49] A. Barenco, C. H. Bennett, R. Cleve, D. P. DiVincenzo, N. Margolus, P. Shor, T. Sleator, J. A. Smolin, and H. Weinfurter, Elementary gates for quantum computation, Phys. Rev. A 52, 3457 (1995).

[50] G. Cybenko, Reducing quantum computations to elementary unitary operations, Comput. Sci. Eng. 3, 27 (2001).
[51] J. F. Cornwell, Group Theory in Physics, Vol. II (Academic, London, 1984).

[52] T. Tilma and E. C. G. Sudarshan, Generalized Euler angle parameterization for $\mathrm{U}(n)$ with applications to $\mathrm{SU}(n)$ coset volume measures, J. Geom. Phys. 52, 263 (2004).

[53] W.-M. Zhang, D. H. Feng, and R. Gilmore, Coherent states: Theory and some applications, Rev. Mod. Phys. 62, 867 (1990). 